\title{
Análise da Revisão Cochrane: Inibidores da Enzima de Conversão da Angiotensina Versus Antagonistas do Receptor da Angiotensina como Prevenção Cardiovascular na Hipertensão Essencial. Cochrane Database Syst Rev. 2014,8: CD009096.
}

\author{
Analysis of the Cochrane Review: Angiotensin Converging Enzyme Inhibitors \\ Versus Angiotensin Receptor Blockers for Primary Hypertension. Cochrane \\ Database Syst Rev. 2014,8: CD009096.
}

Luís NOGUEIRA-SILVA $\rrbracket^{1,2}$, João A. FONSECA ${ }^{2,3}$

Acta Med Port 2015 May-Jun;28(3):283-285

\section{RESUMO}

Os inibidores da enzima de conversão da angiotensina e os antagonistas do receptor da angiotensina são fármacos de primeira linha no tratamento da hipertensão arterial. O objectivo desta revisão foi avaliar se existem diferenças entre estas classes farmacológicas na prevenção de mortalidade total, ocorrência de eventos cardiovasculares e ocorrência de efeitos adversos. Foi realizada uma revisão sistemática com metanálise, pesquisando estudos que comparassem directamente inibidores da enzima de conversão da angiotensina e antagonistas do receptor da angiotensina em diversas bases de dados até Julho de 2014. A selecção dos estudos e extracção de dados foram realizadas por dois investigadores de forma independente. Foram incluídos nove estudos, com um total de 10963 participantes, dos quais 9398 provêm do mesmo estudo, que incluiu apenas participantes com alto risco cardiovascular. Não se verificaram diferenças quanto a mortalidade total, quanto a mortalidade de causa cardiovascular, nem quanto a eventos cardiovasculares totais. Verificou-se um risco ligeiramente menor para os antagonistas do receptor da angiotensina quanto à exclusão de participantes por efeitos adversos (necessário tratar 55 pessoas com antagonistas do receptor da angiotensina durante 4,1 anos para evitar uma exclusão por efeito adverso), principalmente devido à ocorrência de tosse seca com os inibidores da enzima de conversão da angiotensina. Assim, não se encontraram diferenças entre inibidores da enzima de conversão da angiotensina e antagonistas do receptor da angiotensina na prevenção de mortalidade total e eventos cardiovasculares, tendo os antagonistas do receptor da angiotensina apresentado melhor tolerabilidade. Dada a grande proporção de participantes com alto risco cardiovascular, a generalização destes resultados a outras populações é limitada.

Palavras-chave: Antagonistas dos Receptores da Angiotensina; Hipertensão; Inibidores da Enzima de Conversão da Angiotensina; Revisão Sistemática.

\section{ABSTRACT}

Angiotensin converting enzyme inhibitors and angiotensin receptor blockers are first line drugs in the treatment of hypertension. The aim of this review was to assess if there are differences between these drug classes regarding the prevention of total mortality, occurrence of cardiovascular events and of adverse effects. A systematic review and metanalysis was performed, searching for studies that compare angiotensin converting enzyme inhibitors and angiotensin receptor blockers face-to-face, in several databases until July 2014. The study selection and data extraction were performed by 2 independent researchers. Nine studies were included, with a total of 10963 participants, 9398 of which participated in the same study and had high cardiovascular risk. No differences were observed regarding total mortality, cardiovascular mortality or total cardiovascular events. A slightly smaller risk was observed with angiotensin receptor blockers regarding withdrawal due to adverse effects ( 55 people were needed to be treated with angiotensin receptor blockers for 4.1 years to avoid one withdrawal due to adverse effect), mainly due to the occurrence of dry cough with angiotensin converting enzyme inhibitors. Thus, no differences were observed between angiotensin converting enzyme inhibitors and angiotensin receptor blockers in the prevention of total mortality and cardiovascular events, and angiotensin receptor blockers were better tolerated. Given the large proportion of participants with a high cardiovascular risk, the generalization of these results to other populations is limited.

Keywords: Angiotensin-Converting Enzyme Inhibitors; Angiotensin Receptor Antagonists; Hypertension; Systematic Reviews.

\section{INTRODUÇÃO}

A hipertensão arterial (HTA) é um dos principais factores de risco para a ocorrência de eventos cardiovasculares, como o acidente vascular cerebral e o enfarte agudo do miocárdio. Os inibidores da enzima de conversão da an- giotensina (iECAs) e os antagonistas do receptor da angiotensina (ARAs) são fármacos de primeira linha no tratamento da HTA. É frequentemente assumido que estes fármacos são equivalentes dado que são inibidores do eixo

1. Serviço de Medicina Interna. Centro Hospitalar S. João, E.P.E. Porto. Portugal.

2. Centro de Investigação em Tecnologias e Sistemas de Informação em Saúde. Faculdade de Medicina. Universidade do Porto. Porto. Portugal.

3. Unidade de Imunoalergologia. Hospital e Instituto CUF Porto. Porto. Portugal.

$\bowtie$ Autor correspondente: Luís Nogueira-Silva. luisnogueirasilva@gmail.com

Recebido: 01 de Junho de 2015 - Aceite: 01 de Junho de 2015 | Copyright $\odot$ Ordem dos Médicos 2015 
renina-angiotensina-aldosterona, embora o façam em 2 pontos diferentes. Duas revisões Cochrane mostraram que estas famílias de antihipertensores têm eficácia semelhante na descida da pressão arterial (PA) ${ }^{1,2}$, o que não implica necessariamente que tenham igual eficácia na prevenção da ocorrência de eventos cardiovasculares.

\section{QUESTÃO CLÍNICA}

Existe diferença entre iECAs e ARAs quanto aos seus efeitos na mortalidade total, ocorrência de eventos cardiovasculares e de exclusão de participantes por efeitos adversos?

\section{METODOLOGIA}

Nesta revisão foram incluídos ensaios clínicos aleatorizados, em doentes com hipertensão controlada ou não controlada, que comparassem directamente iECAs e ARAs, que tivessem protocolo semelhante nos dois braços no que diz respeito à utilização de outros antihipertensores, que tivessem duração de pelo menos um ano e que fossem duplamente ocultados quando incluídos para a avaliação de exclusão de participantes por eventos adversos. Como outcomes primários foram considerados 1) mortalidade de qualquer causa (mortalidade total), 2) mortalidade de causa cardiovascular (combinação de doença coronária e doença cerebrovascular fatais), 3) morbilidade de causa cardiovascular (combinação de doença coronária e doença cerebrovascular não fatais), 4) eventos cardiovasculares totais, incluindo enfarte agudo do miocárdio fatal e não fatal, outros eventos coronários, acidente vascular cerebral fatal e não fatal, morte e internamento por insuficiência cardíaca congestiva. Como outcome secundário foi considerada a exclusão (abandono do estudo) de participantes por efeitos adversos.

Foram pesquisadas as bases de dados Hypertension Group Specialised Register, Cochrane Central Register of Controlled Trials (CENTRAL), Ovid MEDLINE e Ovid EMBASE, World Health Organization International Clinical Trials Registry Platform e Web of Science até Julho de 2014.

A selecção de estudos e extracção de dados foram realizadas por dois dos autores; as discordâncias foram resolvidas por discussão ou com o auxílio de um terceiro investigador quando necessário. Foram avaliados os riscos de vieses.

Os resultados são apresentados como risco relativo (RR) e seu intervalo de confiança a 95\% (IC 95\%), redução de risco absoluta (RRA) e número necessário para tratar para haver benefício adicional (NNT).

\section{RESULTADOS}

Foram incluídos nove estudos para análise qualitativa e oito para análise quantitativa (num estudo não ocorreram eventos e não foram descritas exclusões de participantes por efeitos adversos); foram incluídos 10963 participantes, com idades médias entre os 45 e os 72 anos, proporções semelhantes de indivíduos de sexo feminino e masculino, média de PA na baseline entre 143/87 e 172/102 mmHg. Os iECAs estudados foram o enalapril (dois estudos), ramipril (quatro estudos) e lisinopril (dois estudos); os ARAs estudados foram o telmisartan (três estudos), losartan (um estudo), candesartan (um estudo) e valsartan (três estudos). Um estudo (ONTARGET ${ }^{3}$ de 2008 que compara ramipril versus telmisartan) tem um grande peso na análise dado incluir 9398 participantes.

Três estudos (10 101 participantes) reportaram eventos relativos a mortalidade total, não se tendo verificado diferenças entre iECAs e ARAs (RR 0,98; IC 95\% 0,88 a 1,10 ). Dois estudos (9600 participantes) reportaram eventos relativos a mortalidade de causa cardiovascular, não se tendo verificado diferenças entre iECAs e ARAs (RR 0,98; IC $95 \%$ 0,85 a 1,13). Nenhum estudo reportou eventos relativos a morbilidade de causa cardiovascular. Apenas um estudo reportou eventos cardiovasculares totais, não se tendo verificado diferenças entre iECAs e ARAs (RR 1,07; IC 95\% 0,96 a 1,19). Os oito estudos (10 963 participantes) reportaram a exclusão de participantes por efeitos adversos, tendo-se verificado um menor risco para os ARAs (duração média de 4,1 anos, RR 0,83; IC 95\% 0,74 a 0,93; RRA 1,8\%, NNT 55). Esta diferença deveu-se à ocorrência de tosse com os iECAs; se for realizada análise de sensibilidade, retirando da análise a ocorrência de tosse seca, as restantes diferenças tornam-se não significativas. Contudo, esta análise inclui apenas 51 eventos com iECAs e 49 eventos com ARAs dado que as razões específicas não estavam disponíveis para o estudo ONTARGET. Não se observou heterogeneidade estatisticamente significativa para nenhum dos outcomes acima descritos.

\section{COMENTÁRIO}

Esta revisão mostra que, no que diz respeito à prevenção de mortalidade total e ocorrência de eventos cardiovasculares, fatais ou não fatais, não existe evidência de diferença entre iECAs e ARAs. Esta evidência é descrita como sendo de grau moderado/baixo 4 dado que os dados quanto à mortalidade total e mortalidade cardiovascular provêm de três estudos de boa qualidade mas os dados quanto à ocorrência de eventos cardiovasculares totais provém apenas de um subgrupo de participantes de um estudo.

Por outro lado, na ocorrência de exclusão de participantes por efeitos adversos, os ARAs mostraram ligeira superioridade face aos iECAs, evidência descrita como sendo de grau alto. ${ }^{4}$ Para a análise deste outcome foram excluídos estudos sem ocultamento, pois a falta de ocultamento pode ter enviesado a decisão de excluir participantes. É defendido nesta revisão que a diferença neste outcome entre iECAs e ARAs é devida à ocorrência de tosse seca. Contudo, esta conclusão tem limitações dado que este achado provém de uma análise de sensibilidade post-hoc incluindo apenas os estudos que reportaram especificamente quais os efeitos adversos que levaram à exclusão de participantes, por isso correspondendo apenas a um total de 1363 participantes (100 excluídos por efeitos adversos) visto que o estudo ONTARGET não forneceu estes dados. 
A maior parte dos dados desta revisão provém do estudo ONTARGET ${ }^{3}$, um estudo que compara ramipril e telmisartan e que incluiu apenas participantes com elevado risco cardiovascular (com história de doença cardiovascular, cerebrovascular ou arterial periférica, ou com diabetes mellitus com lesão de órgão-alvo). Assim, a possibilidade de extrapolar os resultados desta revisão para doentes sem estas características é limitada.

Apesar de terem sido incluídos estudos com vários iECAs e vários ARAs, esta revisão não obteve suficiente poder estatístico para comparar os efeitos de diferentes fármacos ou doses.

\section{REFERÊNCIAS}

1. Heran BS, Wong MM, Heran IK, Wright JM. Blood pressure lowering efficacy of angiotensin converting enzyme (ACE) inhibitors for primary hypertension. Cochrane Database Syst Rev. 2008;4:CD003823.

2. Heran BS, Wong MM, Heran IK, Wright JM. Blood pressure lowering efficacy of angiotensin receptor blockers for primary hypertension. Cochrane Database Syst Rev. 2008;4 :CD003822.

\section{IMPLICAÇÕES PARA A PRÁTICA}

Não foram encontradas diferenças no que diz respeito à prevenção de mortalidade e eventos cardiovasculares quando comparados iECAs e ARAs enquanto classes farmacológicas. Os ARAs mostraram ter melhor tolerabilidade, embora de forma ligeira dado que é necessário tratar 55 pessoas com um ARA durante 4,1 anos para prevenir uma exclusão por efeito adverso. Dado que a maior parte dos dados provém de um estudo que incluiu apenas participantes com alto risco cardiovascular, a possibilidade de generalização destas conclusões para outras populações é limitada. São necessários mais dados para que se estabeleça evidência quanto à diferença de iECAs e ARAs noutras populações e quanto à diferença de diferentes fármacos ou doses.
3. ONTARGET Investigators, Yusuf S, Teo KK, Pogue J, Dyal L, Copland I, et al. Telmisartan, ramipril, or both in patients at high risk for vascular events. N Engl J Med. 2008;358:1547-59.

4. Guyatt GH, Oxman AD, Vist GE, Kunz R, Falck-Ytter Y, Alonso-Coello $P$, et al. GRADE: an emerging consensus on rating quality of evidence and strength of recommendations. BMJ. 2008;336:924-6.

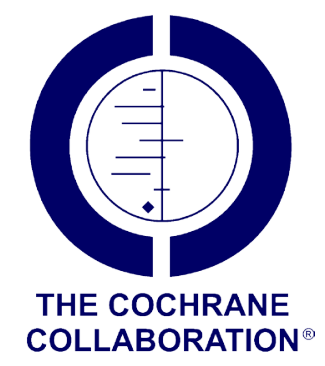


Luís NOGUEIRA-SILVA, João A. FONSECA

\section{Análise da Revisão Cochrane: Inibidores da} Enzima de Conversão da Angiotensina Versus Antagonistas do Receptor da Angiotensina como Prevenção Cardiovascular na Hipertensão Essencial. Cochrane Database Syst Rev. 2014,8: CD009096.

Acta Med Port 2015:28:283-285

Publicado pela Acta Médica Portuguesa, a Revista Científica da Ordem dos Médicos

Av. Almirante Gago Coutinho, 151

1749-084 Lisboa, Portugal.

Tel: +351218428215

E-mail: submissao@actamedicaportuguesa.com

www.actamedicaportuguesa.com

ISSN:0870-399X | e-ISSN: 1646-0758

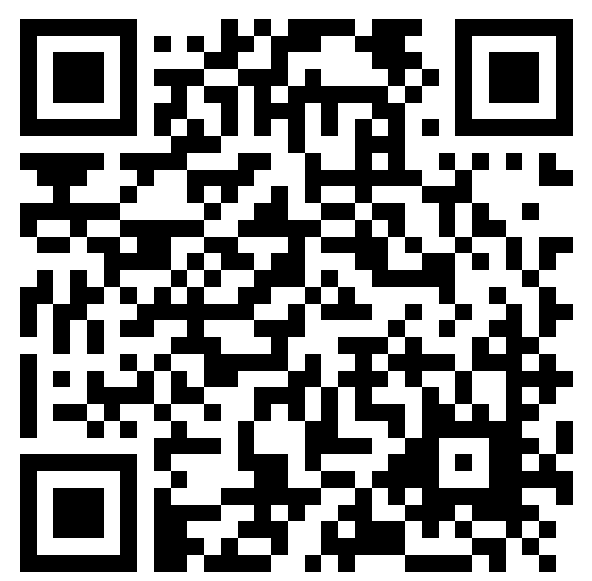

